Theoretical and Mathematical Physics, Vol. 124, No. 3, 2000

\title{
MODEL OF A SPATIALLY INHOMOGENEOUS ONE-DIMENSIONAL ACTIVE MEDIUM
}

\author{
K. A. Vasil'ev, ${ }^{1}$ A. Yu. Loskutov, ${ }^{1}$ S. D. Rybalko, ${ }^{1}$ and D. N. Udin ${ }^{1}$
}

We investigate the dynamics of one-dimensional discrete models of a one-component active medium analytically. The models represent spatially inhomogeneous diffusively concatenated systems of one-dimensional piecewise-continuous maps. The discontinuities (the defects) are interpreted as the differences in the parameters of the maps constituting the model. Two classes of defects are considered: spatially periodic defects and localized defects. The area of regular dynamics in the space of the parameters is estimated analytically. For the model with a periodic inhomogeneity, an exact analytic partition into domains with regular and with chaotic types of behavior is found. Numerical results are obtained for the model with a single defect. The possibility of the occurrence of each behavior type for the system as a whole is investigated.

\section{Introduction}

The approximate representation of continuous media by their discrete analogues is a rather effective research method. The conversion to the discrete form can be either purely spatial or both spatial and temporal. In the spatial discretization, the initial system is approximated by a finite or countable set of clements with a certain form of coupling between them. Every element represents a dynamic system with a small number of variables. If, in addition, the dynamic system is determined by a map, i.e., by a transformation with discrete time, then the discretization is called spatial-temporal. The spatialtemporal discrete models are called net or lattice models. Many problems in the nonlinear theory of a continuous medium can be reduced to problems with discrete models. For example, some problems in statistical physics can be effectively solved through the representation of the medium by its discrete space-time approximation [1-4], excitable continuous systems are often described in terms of their discrete analogues $[4,5]$, etc. (cf. [6-13] and the references therein). Moreover, any sort of numerical analysis of continuous systems is always connected with discrete space-time systems because any numerical procedure is based on a finite difference scheme.

There is a variety of space time lattice forms. The most common are the lattices where every element somehow interacts only with its nearest neighbors (see, e.g., [8-13] and the references therein). Another form of coupling is global interaction in which every pair of elements is connected [14--16]. In the case of a local interaction, diffusion coupling between the elements is normally considered $[1,2,4,8-13]$ (see also the references therein). This form of coupling is mainly used to model the phenomena related to space-time chaos, structure generation, and self-organization.

If the value of a governing parameter of the family of maps constituting a lattice is constant for the entire lattice, the lattice is called homogeneous. Varying this parameter and the value of the diffusion (or the coupling constant), we can investigate the phase diagram, the possible phase transitions, and the related space-time patterns. If the parameters of the maps are different, then the net is inhomogeneous. Such nets are much more difficult to study. Almost all previous works dedicated to diffusively interacting maps

\footnotetext{
${ }^{1}$ Moscow State University, Moscow, Russia.
}

Translated from Teoreticheskaya i Matematicheskaya Fizika, Vol. 124, No. 3, pp. 506-519, September, 2000. Original article submitted September 20, 1999; revised April 13, 2000. 
were restricted to a study (usually a numerical study) of homogeneous nets. From the physical standpoint, however, it is obvious that the space homogeneity (or, in this case, the identity of all elements) is a strong idealization adopted to simplify the analysis. It is therefore interesting to investigate the qualitative changes in the system dynamics related to the inhomogeneity. The latter can be of different types ranging from individual defects to a periodic inhomogeneity of the entire space.

The present work is focused on spatially inhomogeneous one-dimensional systems (i.e., chains) of diffusively connected piecewise-linear one-dimensional maps. The term map here refers to a transformation of an interval $I$ into itself: $T_{a}: I \rightarrow I$, where $I=[\alpha, \beta] \in \mathbb{R}^{1}, x \mapsto G(x, a), x \in I, G(x, a)$ is a function, and $a$ is a parameter (or, generally, a set of parameters). In terms of the iterations $k$, this can be written as $x(k+1)=G(x(k), a), x \in I$. The inhomogeneities are given as maps with different parameters. The maps themselves (i.e., the functions $G$ ) are chosen such that the systems represent models of a one-component active medium [17]. Based on an exact calculation of the Lyapunov indices, we investigate different regimes in the behavior of the system with a periodic inhomogeneity and of the system with a single defect. The phase space structure of these systems is also described.

\section{Models of spatially inhomogeneous active media}

The main result in this section is the description of the dynamics of two types of inhomogeneous active media. We consider a model representing a one-dimensional annular lattice of concatenated maps with a periodic inhomogeneity and a chain with a single defect.

2.1. Homogeneous medium. We examine a system of $N$ diffusively concatenated maps of the form

$$
x_{n}(k+1)=G\left(x_{n}(k), \alpha, \gamma\right)+\varepsilon\left(x_{n+1}(k)-2 x_{n}(k)+x_{n-1}(k)\right)
$$

with the periodic boundary conditions $x_{n+N}(k) \equiv x_{n}(k)$, where $n=1,2, \ldots, N$ is the discrete space coordinate, $k=0,1,2, \ldots$ is the discrete time coordinate, $\varepsilon>0$ is the diffusion coefficient (which we assume to be constant), and $\alpha>0$ and $\gamma$ are parameters. For the function $G$, we set

$$
G(x, \alpha, \gamma)=x+\alpha F(x)-\gamma= \begin{cases}(1-2 \alpha) x-\gamma, & x<1 / 2 \\ (1-2 \alpha) x-\gamma+2 \alpha, & x>1 / 2 .\end{cases}
$$

This form of the function $G(x, \alpha, \gamma)$ was chosen because system of maps (1) with elements of form (2) is a discrete realization of the basic model of a one-component one-dimensional active medium described by an equation of the Kolmogorov-Petrovskii-Piskunov form:

$$
\frac{\partial U(x, t)}{\partial t}=D \frac{\partial^{2} U(x, t)}{\partial x^{2}}+\Phi(U) .
$$

As is known, this equation is commonly met in problems in biophysics, combustion theory, chemical kinetics (e.g., the description of the Belousov-Zhabotinskii reaction), solid state electronics, etc. (see, e.g., [18-21]).

It is easy to see that $G(x, \alpha, \gamma)$ is a piecewise-linear function whose graph has a constant slope equal to $(1-2 \alpha)$. Depending on the parameter $\alpha$, the map generated by the function $G$, i.e., $x \mapsto G(x, \alpha, \gamma)$, can manifest qualitatively different types of behavior. For $0<\alpha<1$ (Fig. 1a), its behavior is regular, and depending on $\gamma$, the map can have one or two stable points (the points $A$ and $B$ ) attracting almost all points of the phase space. For $\alpha>1$, the absolute value of the slope of the graph of the map exceeds unity (see Fig. 1b), which corresponds to an exponential divergence of neighboring trajectories. In this case, if the motion of a phase point is finite, the dynamies is chaotic.

Homogeneous model (1) composed of concatenated functions of form (2) was described in detail in [17]. We recall the principal results of this study. System (1), (2) can be interpreted as an $N$-dimensional 


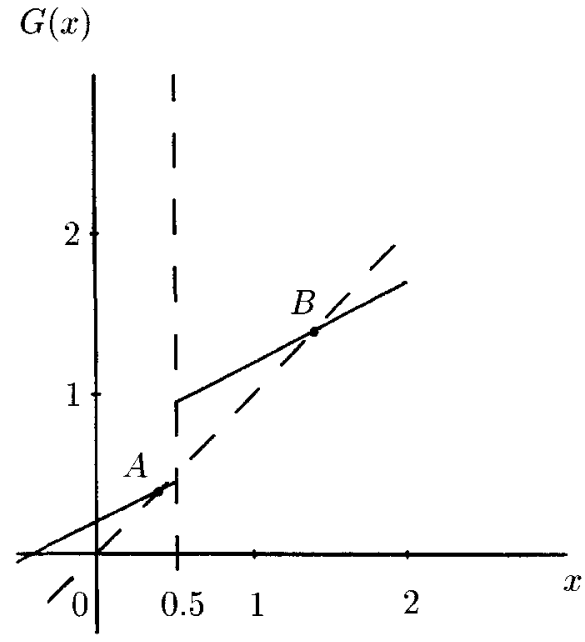

(a)

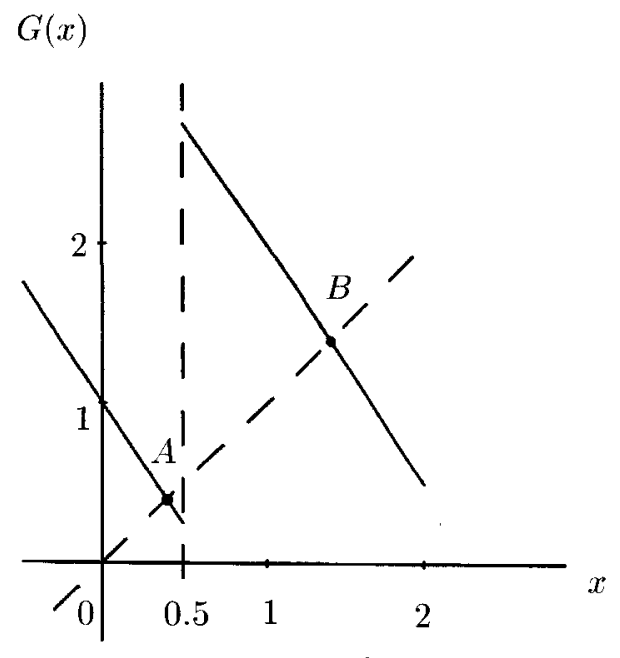

(b)

Fig. 1. The map $x \mapsto G(x, \alpha, \gamma)$ : (a) for $\alpha=0.25$ and $\gamma=-0.2$; (b) for $\alpha=125$ and $\gamma=-1$.

piecewise-linear map $f: \mathbb{R}^{N} \rightarrow \mathbb{R}^{N}$. Because $G(x, \alpha, \gamma)$ is a piecewise-linear function with a constant derivative, the differential $\mathrm{D}_{f}$ of the map $f$ is a matrix with constant coefficients. It is therefore easy to find the Lyapunov indices $\lambda_{i}$ of this map. If $\rho_{s}$ are the eigenvalues of $D_{f}$, then $\lambda_{s}=\log \left|\rho_{s}\right|$. If any eigenvalue $\rho_{s}$ lies outside the unit circle on the complex plane, then all trajectories of the map $f$ are unstable. Othorwise, the model dynamics is regular. The problem of the system behavior is therefore connected with investigating the location of the roots $\rho_{s}$. To calculate $\rho_{s}$, we express the characteristic polynomial for the $N$-dimensional map differential in terms of determinants of three-diagonal matrices with different dimensions. We can obtain recursive relations for these matrices. Interpreting these relations as finitedifference equations with given initial conditions, it is possible to find the solutions, which are proportional to the Chebyshev polynomials of a linear function of the eigenvalues of $\mathrm{D}_{f}$. It can be shown that because of the properties of Chebyshev polynomials, the characteristic polynomial reduces to a quadratic third-order polynomial of the known function $\rho_{s}$. Calculating the values of $\rho_{s}$ and analyzing their locations on the complex plane lead to a partition of the parameter space of map system (1), (2) into two domains (Fig. 2):

1. Domain $D_{1}$ is determined by parameters $\alpha$ and $\varepsilon$ such that the absolute values of all eigenvalues of the map $f$ differential are smaller than unity, and the model dynamics is therefore regular.

2. Domain $\mathrm{D}_{2}$ is determined through the condition that the set of eigenvalues $\rho_{s}$ contains at least one root satisfying the inequality $\left|\rho_{s}\right|>1$. In this case, generally, the dynamics can be infinite. However, if the motion is finite, the behavior of system (1), (2) is said to be chaotic [9-11].

System (1), (2) is spatially homogeneous in the sense that all its elements (i.e., the maps $x \mapsto G(x, \alpha, \gamma)$ ) are equal. However, our goal is to investigate the spatially inhomogeneous diffusive model. The inhomogeneity can be related either to different map types acting in different space points or (if the maps for all the elements are equal) to different map parameters.

In this analytic study, we restrict ourselves to the latter case, i.e., we assume that every element is a piecewise-linear map $x_{i} \mapsto G\left(x_{i}, \alpha_{i}, \gamma\right)$ (see Eq. (2)) but, in contrast to the homogeneous case, there are two element types distinguished by the values of the parameters $\alpha_{i}$ of the function $G\left(x_{i}, \alpha_{i}, \gamma\right) .^{2}$ We let the parameter $\alpha$ correspond to one type of the elements and the parameter $\beta$ correspond to the other type.

\footnotetext{
${ }^{2}$ Generally, the elements can also differ by the value of $\gamma$. However, it is shown below that the dynamics of both the homogeneous and the inhomogeneous systems does not depend on $\gamma$.
} 


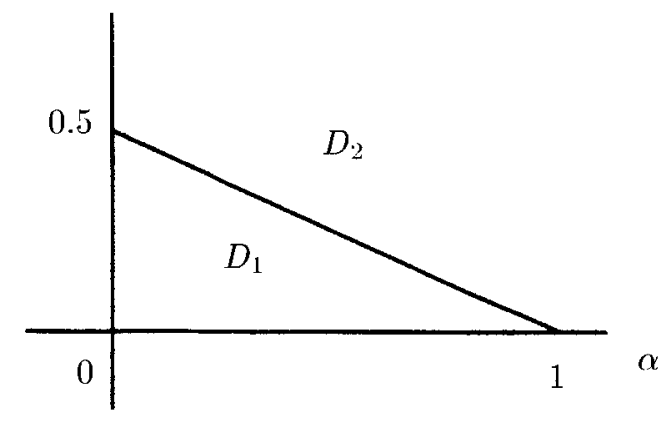

Fig. 2. Parameter space of a homogeneous annular chain of diffusively concatenated maps (1), (2).

As in homogeneous case (1), we take the chain in the form of a ring, i.e., $x_{n+N}(k) \equiv x_{n}(k)$.

We investigate two qualitatively different cases in detail: the model with spatially periodic defects and the model with a single defect.

2.2. Annular model with a spatially periodic inhomogeneity. The term spatially periodic inhomogeneity refers to the case where the values of the parameter of the function $G$ change from one element to another periodically, i.e., the parameters vary as $\alpha, \beta, \alpha, \beta, \ldots$. The dynamics of a chain with such an inhomogeneity can be fully described analytically.

We consider a concatenated one-dimensional net of the form

$$
x_{n}(k+1)= \begin{cases}G\left(x_{n}(k), \alpha, \gamma\right)+\varepsilon\left(x_{n+1}(k)-2 x_{n}(k)+x_{n-1}(k)\right) & \text { for odd } n, \\ G\left(x_{n}(k), \beta, \gamma\right)+\varepsilon\left(x_{n+1}(k)-2 x_{n}(k)+x_{n-1}(k)\right) & \text { for even } n,\end{cases}
$$

where $x_{n+N}(k) \equiv x_{n}(k)$, the function $G$ is still given by relation (2), and $N$ is even (otherwise chain (3) cannot be converted to the form of a ring). We seek the values of the parameters $\alpha, \beta$, and $\varepsilon$ corresponding to qualitatively different regimes of behavior of periodically inhomogeneous annular model (3), (2). We calculate the Lyapunov indices of system (3), (2) using the technique developed in [17].

Taking the boundary conditions into account, we express the differential of the $N$-dimensional map generated by a system of form (3) as

$$
\mathrm{D}_{f} \equiv Q_{N}=\left(\begin{array}{ccccc}
1-2 \varepsilon-2 \alpha & \varepsilon & 0 & \cdots & \varepsilon \\
\varepsilon & 1-2 \varepsilon-2 \beta & \varepsilon & \cdots & 0 \\
0 & \varepsilon & 1-2 \varepsilon-2 \alpha & \cdots & 0 \\
\vdots & \vdots & \vdots & \ddots & \vdots \\
\varepsilon & 0 & 0 & \cdots & 1-2 \varepsilon-2 \beta
\end{array}\right) .
$$

To find the eigenvalues $\rho_{s}, s=1,2, \ldots, N$, of the matrix $Q_{N}$, we calculate the determinant

$$
\operatorname{det}\left(Q_{N}-\rho I_{N}\right)=\left|\begin{array}{cccccc}
2 z_{1} \varepsilon & \varepsilon & 0 & 0 & \cdots & \varepsilon \\
\varepsilon & 2 z_{2} \varepsilon & \varepsilon & 0 & \cdots & 0 \\
0 & \varepsilon & 2 z_{1} \varepsilon & \varepsilon & \cdots & 0 \\
\vdots & \vdots & \vdots & \vdots & \ddots & \vdots \\
0 & 0 & 0 & 0 & \cdots & \varepsilon \\
\varepsilon & 0 & 0 & 0 & \cdots & 2 z_{2} \varepsilon
\end{array}\right|,
$$


where $z_{1}$ and $z_{2}$ are determined from the relations $1-2 \varepsilon-2 \alpha-\rho=2 z_{1} \varepsilon$ and $1-2 \varepsilon-2 \beta-\rho=2 z_{2} \varepsilon$. Expanding the determinant with respect to the first row, we obtain

$$
\operatorname{det}\left(Q_{N}-\rho I_{N}\right)=2 z_{1} \varepsilon B_{N-1}-\varepsilon^{2} B_{N-2}-2 \varepsilon^{N}-\varepsilon^{2} \widehat{B}_{N-2}
$$

where

$$
B_{N}=\left|\begin{array}{ccccc}
2 z_{1} \varepsilon & \varepsilon & 0 & \cdots & 0 \\
\varepsilon & 2 z_{2} \varepsilon & \varepsilon & \vdots & 0 \\
0 & \varepsilon & 2 z_{1} \varepsilon & \cdots & 0 \\
\vdots & \vdots & \vdots & \ddots & \vdots \\
0 & 0 & 0 & \cdots & 2 z_{2} \varepsilon
\end{array}\right|, \quad \widehat{B}_{N}=\left|\begin{array}{ccccc}
2 z_{2} \varepsilon & \varepsilon & 0 & \cdots & 0 \\
\varepsilon & 2 z_{1} \varepsilon & \varepsilon & \cdots & 0 \\
0 & \varepsilon & 2 z_{2} \varepsilon & \cdots & 0 \\
\vdots & \vdots & \vdots & \ddots & \vdots \\
0 & 0 & 0 & \cdots & 2 z_{1} \varepsilon
\end{array}\right| .
$$

It is easy to see that $\widehat{B}_{N}\left(z_{1}, z_{2}\right)$ can be obtained from $B_{N}\left(z_{1}, z_{2}\right)$ through the substitution $z_{1} \leftrightarrow z_{2}$, and we therefore use a recursive relation to find $B_{N}\left(z_{1}, z_{2}\right)$. Expanding $B_{N}$ with respect to the first row, we obtain

$$
B_{N}= \begin{cases}2 z_{1} \varepsilon B_{N-1}-\varepsilon^{2} B_{N-2} & \text { for even } N \\ 2 z_{2} \varepsilon B_{N-1}-\varepsilon^{2} B_{N-2} & \text { for odd } N\end{cases}
$$

It is now easy to express the odd-order determinants through the even-order determinants:

$$
B_{2 N+1}=\frac{B_{2 N+2}+\varepsilon^{2} B_{2 N}}{2 z_{1} \varepsilon}
$$

We then obtain a recursive relation for the even-order determinants:

$$
B_{2 N}=\varepsilon^{2}\left(4 z_{1} z_{2}-2\right) B_{2 N-2}-\varepsilon^{4} B_{2 N-4}
$$

Relation (5) can be interpreted as a difference equation with the initial conditions

$$
B_{0}=1, \quad B_{2}=\varepsilon^{2}\left(4 z_{1} z_{2}-1\right)
$$

Further, solving system (5), (6) and taking Eq. (4) into account, we obtain

$$
B_{N}\left(z_{1}, z_{2}\right)= \begin{cases}\varepsilon^{N} U_{N}(z) & \text { for even } N, \\ \varepsilon^{N} \sqrt{\frac{z_{2}}{z_{1}}} U_{N}(z) & \text { for odd } N\end{cases}
$$

where $U_{N}(z)$ is a Chebyshev polynomial of the second kind. In turn, the relations for $\widehat{B}_{N}\left(z_{1}, z_{2}\right)$ have the form

$$
\widehat{B}_{N}\left(z_{1}, z_{2}\right)= \begin{cases}\varsigma^{N} U_{N}(z) & \text { for even } N \\ \varsigma^{N} \sqrt{\frac{z_{1}}{z_{2}}} U_{N}(z) & \text { for odd } N .\end{cases}
$$

Using the relations for $B_{N-1}, B_{N-2}$, and $\widehat{B}_{N-2}$, we find

$$
\operatorname{det}\left(Q_{N}-\rho I_{N}\right)=2 \varepsilon^{N}\left(z U_{N-1}(z)-U_{N-2}(z)-1\right) .
$$


It is now easy to find the eigenvalues of the differential of map (3), (2). It follows from Eq. (7) that

$$
z U_{N-1}(z)-U_{N-2}(z)-1=0
$$

or $T_{N}(z)-1=0$, where we use the relation between the second-kind Chebyshev polynomials $U_{N}(z)$ and the first-kind Chebyshev polynomials $T_{N}(z)$. Because

$$
T_{N}(z)=\frac{\left(z-\sqrt{z^{2}-1}\right)^{N}+\left(z-\sqrt{z^{2}-1}\right)^{-N}}{2}
$$

(where $z^{2}=z_{1} z_{2}$ ), if we set $t=z-\sqrt{z^{2}-1}$, we obtain

$$
\frac{1}{2}\left(t^{N}+\frac{1}{t^{N}}\right)-1=0
$$

or $\left(t^{N}-1\right)^{2}=0$ from Eq. (8). Consequently, $t_{s}=e^{i 2 \pi s / N}$ and $z_{s}=\cos (2 \pi s / N), s=1,2, \ldots, N$. Recalling that $2 z_{1} \varepsilon \cdot 2 z_{2} \varepsilon=4 z^{2} \varepsilon^{2}$, we obtain the simple equation

$$
(1-2 \varepsilon-2 \alpha-\rho)(1-2 \varepsilon-2 \beta-\rho)=4 \varepsilon^{2} \cos ^{2}\left(\frac{2 \pi}{N} s\right) .
$$

Solving this equation with respect to $\rho$, we find the set of eigenvalues of the differential of map (3), (2):

$$
\rho_{1,2}^{s}=1-2 \varepsilon-\alpha-\beta \pm \sqrt{(\alpha-\beta)^{2}+4 \varepsilon^{2} \cos ^{2}\left(\frac{2 \pi}{N} s\right)},
$$

where $s=1,2, \ldots, N / 2$. The dynamics of system (3), (2) is fully regular if all values of $\rho_{s}$ lie inside the unit circle on the complex plane. All $\rho_{1,2}^{s}$ are real; therefore, the regular-dynamics condition is satisfied if

$$
\begin{aligned}
& 2 \varepsilon+\alpha+\beta>\sqrt{4 \varepsilon^{2}+(\alpha-\beta)^{2}}, \\
& \varepsilon+\frac{\alpha+\beta}{2}+\sqrt{\varepsilon^{2}+\left(\frac{\alpha-\beta}{2}\right)^{2}}<1 .
\end{aligned}
$$

Because $\varepsilon, \alpha, \beta>0$, the first inequality of this system is always true. In turn, the condition $\varepsilon+(\alpha+\beta) / 2+$ $\sqrt{\varepsilon^{2}+(\alpha-\beta)^{2} / 4}>1$ is satisfied in the parameter space domain where $\lambda_{s}>0$.

The boundary between the domains of qualitatively different (regular or chaotic) dynamics is a surface in the three-dimensional space of the parameters $(\alpha, \beta, \varepsilon)$ given by the expression

$$
\varepsilon+\frac{\alpha+\beta}{2}+\sqrt{\varepsilon^{2}+\frac{(\alpha-\beta)^{2}}{4}}=1 .
$$

As an illustration, we consider the sections of the phase space of system (3), (2) by planes $\delta=$ const, where $\delta=\beta-\alpha$ is the inhomogeneity parameter (Fig. 3). Let $D_{1}$ be the regular-dynamics domain, and let $D_{2}$ be the domain where $\lambda_{s}>0$. The boundary between these two domains is determined by an equation that follows from Eq. (10). For comparison, the boundaries separating the domains $D_{1}$ and $D_{2}$ for the homogeneous $(\delta=0)$ system of concatenated maps (see Fig. 2) are shown in Fig. 3a,b (dashed lines). As follows from Fig. 2, the model with $\delta>1$ exhibits a chaotic dynamics for any values of $\varepsilon$ and $\alpha$. For $\delta<1$, there exists a domain $D_{1}$ where the dynamics is regular. In a certain range of values of the parameters $0<\delta<1$, this domain is a subarea of the regular behavior domain of the homogeneous model. For 


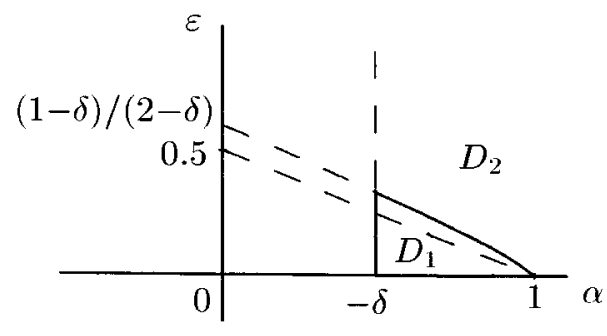

(a)

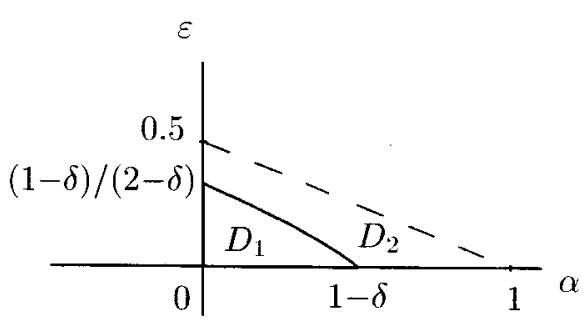

(b)

Fig. 3. Sections of the parameter space for model (2), (3) by the planes $\delta=$ const: (a) for $-1<\delta<0$; (b) for $0<\delta<1$.

$-1<\delta<0$, there exist values of the parameters $\varepsilon$ and $\alpha$ such that the dynamics of the inhomogeneous model is regular, while the dynamics of the homogeneous one is chaotic. The restriction $\alpha>-\delta$ corresponds to the condition $\beta>0$. For $\delta<-1$, the domain $D_{1}$ vanishes, and model (3), (2) exhibits only chaotic properties for all possible values of $\varepsilon$ and $\alpha$. As directly follows from Fig. 3 , there are two qualitatively different possibilities: either the parameter $\alpha$ belongs to the regularity domain, the parameter $\beta$ belongs to the chaos domain with $\lambda_{s}>0$, and the general dynamics is regular or the dynamics of the periodically inhomogeneous model can be chaotic. The realization of one of these two possibilities depends on the value of the diffusion parameter $\varepsilon$.

2.3. Annular model with a single defect. We now consider another interesting spatially inhomogeneous model of diffusively concatenated maps, the system with a single defect. In this case, the parameter $\beta$ (see formula (2)) corresponds to one of the $N$ elements of the system, while the parameter $\alpha$ corresponds to the remaining $N-1$ elements. Without loss of generality, we can set this single different element to correspond to $n=1$. Therefore, we examine a model of the form

$$
x_{n}(k+1)= \begin{cases}G\left(x_{n}(k), \alpha, \gamma\right)+\varepsilon\left(x_{n-1}(k)-2 x_{n}(k)+x_{n+1}(k)\right), & n=2,3, \ldots, N, \\ G\left(x_{1}(k), \beta, \gamma\right)+\varepsilon\left(x_{N}(k)-2 x_{1}(k)+x_{2}(k)\right), & n=1,\end{cases}
$$

where $x_{n+N} \equiv x_{n}(k)$. As above, let the function $G(x, \alpha, \gamma)$ be given by expression (2). We determine the differential of the corresponding map for system $(11),(2)$ :

$$
\mathrm{D}_{f} \equiv Q_{N}=\left(\begin{array}{ccccc}
1-2 \varepsilon-2 \beta & \varepsilon & 0 & \cdots & \varepsilon \\
\varepsilon & 1-2 \varepsilon-2 \alpha & \varepsilon & \cdots & 0 \\
0 & \varepsilon & 1-2 \varepsilon-2 \alpha & \cdots & 0 \\
\vdots & \vdots & \vdots & \ddots & \vdots \\
\varepsilon & 0 & 0 & \cdots & 1-2 \varepsilon-2 \alpha
\end{array}\right) .
$$

If the technique used above to find the Lyapunov indices (see Secs. 2.1 and 2.2 and also [17]) is applied to model (11), (2), then the characteristic equation for map differential (12) leads to the necessity to find the roots of a polynomial of order $2 N+2$. This can only be done numerically. We therefore use a somewhat different approach for this model.

We estimate the regular-dynamics domain of system (11), (2) using the estimate for the eigenvalues of matrix (12) based on the Guershgorin theorem [22]. According to this theorem, all eigenvalues of a matrix $A=\left\{a_{i j}\right\}_{n \times n}$ belong to a union of circles on the complex plane:

$$
\rho_{s} \in \bigcup_{i=1}^{n}\left\{z \in \mathbb{C}: \| z-a_{i j} \mid \leq R_{i}^{\prime}\right\}, \quad s=1,2, \ldots, N
$$




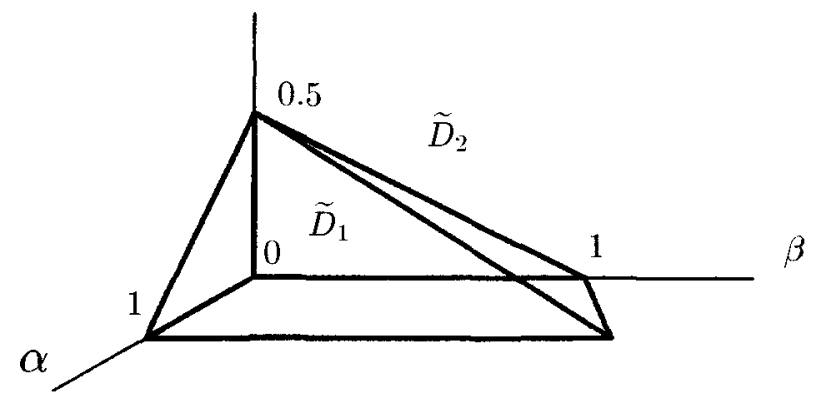

Fig. 4. Estimate of the regular-dynamics domain for inhomogeneous chains with two element types in the parameter space $(\alpha, \beta, \varepsilon)$.

where $R_{i}^{\prime}$ is either the row quasi-norm of the matrix $A, R_{i}^{\prime}=\sum_{j=1, j \neq i}^{n}\left|a_{i j}\right|$, or the columnar quasi-norm of the matrix $A, R_{j}^{\prime}=\sum_{i=1, i \neq j}^{n}\left|a_{i j}\right|$. Because map differential (12) is a real symmetric matrix, the row and the columnar quasi-norms coincide, and the eigenvalues of $D_{f}$ are real. Therefore, the Guershgorin circles are transformed into intervals on the real axis:

$$
\rho_{s} \in \bigcup_{i=1}^{n}\left\{z \in \mathbb{R}:\left|z-a_{i j}\right| \leq R_{i}^{\prime}\right\}, \quad s=1,2, \ldots, N .
$$

Because $\varepsilon>0$, it is easy to find from expression (12) that

$$
R_{i}^{\prime}=\sum_{\substack{j=1 \\ j \neq i}}^{n}\left|a_{i j}\right|=|\varepsilon|+|\varepsilon|=2 \varepsilon .
$$

We note that $R_{i}^{\prime}$ does not depend on $i$ and there are only two different types of diagonal elements in the map differential $D_{f}$ (see Eq. (12)); therefore, the eigenvalues of $\mathrm{D}_{f}$ belong to the union of two intervals

$$
\{z \in \mathbb{R}:|1-2 \varepsilon-2 \alpha-z| \leq 2 \varepsilon\} \cup\{z \in \mathbb{R}:|1-2 \varepsilon-2 \beta-z| \leq 2 \varepsilon\} .
$$

Because $\alpha, \beta, \varepsilon>0$, we then obtain an upper estimate for the absolute values of the eigenvalues of $\mathrm{D}_{f}$ :

$$
\left|\rho_{s}\right| \leq \begin{cases}\beta+2 \varepsilon, & \alpha<\beta \\ \alpha+2 \varepsilon, & \alpha>\beta\end{cases}
$$

Therefore, the domain $\widetilde{D}_{1}$ in the parameter space $(\alpha, \beta, \varepsilon)$ satisfying the conditions

$$
\widetilde{D}_{1}: \quad \begin{cases}\beta+2 \varepsilon<1 & \text { for } \alpha<\beta \\ \alpha+2 \varepsilon<1 & \text { for } \alpha>\beta\end{cases}
$$

corresponds to a regular dynamics of inhomogeneous model (11), (2), and the domain

$$
\widetilde{D}_{2}: \begin{cases}\beta+2 \varepsilon>1 & \text { for } \alpha<\beta \\ \alpha+2 \varepsilon>1 & \text { for } \alpha>\beta\end{cases}
$$

corresponds to positive $\lambda_{s}$. The domains $\widetilde{D}_{1}$ and $\widetilde{D}_{2}$ are shown in Fig. 4. The domain $\widehat{D}_{1}$ is a lower estimate for the region of regular dynamics, because it was derived using the upper estimate for the eigenvalues of 


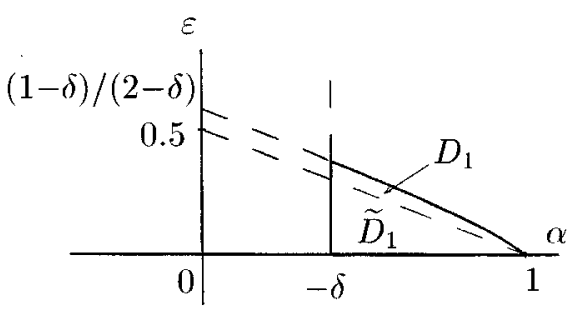

(a)

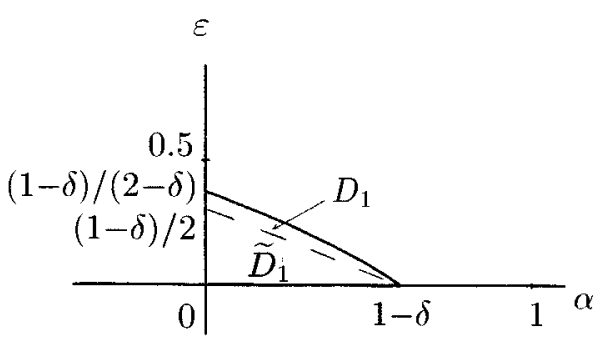

(b)

Fig. 5. Approximate estimate and exact result for the regular-dynamics domain of the model with periodic inhomogeneity: (a) for $-1<\delta<0$; (b) for $0<\delta<1$.

the map differential. Any point of the parameter space belonging to $\widetilde{D}_{1}$ corresponds to a regular dynamics of the model, while any point belonging to $\widetilde{D}_{2}$ corresponds to either regular or (if the motion is finite) chaotic dynamics.

The derived estimate is valid not only for the single-defect model discussed but also for the entire class of annular systems of maps with a diffusive concatenation of form (1) characterized by the presence of two types of the elements (with different parameters $\alpha_{i}$ of the function $G\left(x_{i}, \alpha_{i}, \gamma\right)$ ). In this case, the number of elements of each type and their relative positions do not matter. Indeed, the quasi-norms of the map differentials for all such systems coincide, and the centers of two possible intervals containing the eigenvalues of $\mathrm{D}_{f}$ (according to the Guershgorin theorem) are common as well.

By a corollary of the Guershgorin theorem, if the two intervals where the estimate of $\rho_{s}$ is made do not intersect, then there is a "clustering" of the map differential cigenvalues. That is, $q$ eigenvalues $\rho_{s}$, $s=1,2, \ldots, q$, lie within one interval, and $N-q$ eigenvalues $\rho_{s}, s=q+1, q+2, \ldots, N$, lie within the other interval. Here, $q$ is the number of elements with the parameter $\alpha$, and $N-q$ is the number of elements with the parameter $\beta$. However, taking this property into account does not improve the estimate of the regular-dynamics domain.

We compare estimate (13) with the exact result obtained above for the annular model with a spatially periodic inhomogeneity (see Sec. 2.2). It is clear that the model belongs to the class for which this estimate remains valid. For elucidation, we construct the sections $\delta=$ const of the domain $D_{1}$ corresponding to a regular dynamics of system (3), (2) and also those for the estimate $\widetilde{D}_{1}$ (Fig. 5). It can be seen in the figure that estimate (13) for the regular-dynamics domain approximates the true behavior of the model rather well. However, the estimate fails to reflect that for $-1<\delta<0$, the regular-dynamics domain for system (3), (2) is broader than the regular-dynamics domain of the spatially homogeneous system.

We now return to system of maps (11), (2) whose spatial inhomogeneity is connected with the presence of only one defect. We find the eigenvalues of map differential (12) numerically. In this case, the characteristic polynomial can be written as

$$
\operatorname{det}\left(Q_{N}-\rho I_{N}\right)=\left|\begin{array}{ccccc}
2 z \varepsilon+2(\alpha-\beta) & \varepsilon & 0 & \cdots & \varepsilon \\
\varepsilon & 2 z \varepsilon & \varepsilon & \cdots & 0 \\
0 & \varepsilon & 2 z \varepsilon & \cdots & 0 \\
\vdots & \vdots & \vdots & \ddots & \vdots \\
\varepsilon & 0 & 0 & \cdots & 2 z \varsigma
\end{array}\right|,
$$

where $2 z \varepsilon=1-2 \varepsilon-2 \alpha-\rho$. Expanding $\operatorname{det}\left(Q_{N}-\rho I_{N}\right)$ with respect to the first row, we obtain

$$
\operatorname{det}\left(Q_{N}-\rho I_{N}\right)=[2 z \varepsilon+2(\alpha-\beta)] B_{N-1}-2 \varepsilon^{2} B_{N-2}-2(-1)^{N} \varepsilon^{N}
$$




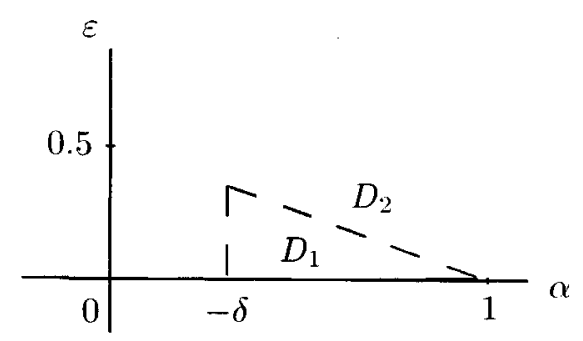

(a)

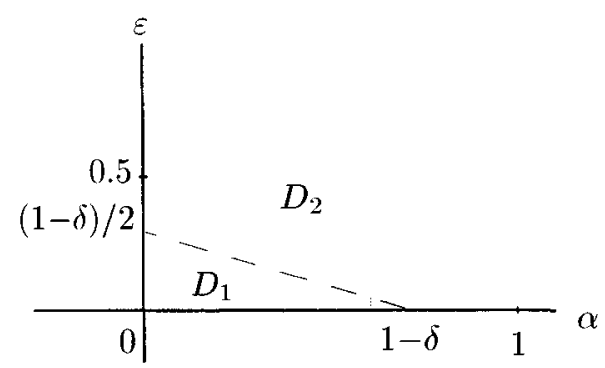

(b)

Fig. 6. Characteristic form of the sections $\delta=$ const of the parameter space for a chain of $N=20$ elements with a single defect: (a) for $\delta=-0.3$; (b) for $\delta=0.3$.

where

$$
B_{N}=\left|\begin{array}{ccccc}
2 z \varepsilon & \varepsilon & 0 & \cdots & 0 \\
\varepsilon & 2 z \varepsilon & \varepsilon & \cdots & 0 \\
0 & \varepsilon & 2 z \varepsilon & \cdots & 0 \\
\vdots & \vdots & \vdots & \ddots & \vdots \\
0 & 0 & 0 & \cdots & 2 z \varepsilon
\end{array}\right|
$$

As above, we use the expansion of $B_{N}$ with respect to the first row to obtain the recursive relation $B_{N}=$ $2 z \subseteq B_{N-1}-\varepsilon^{2} B_{N-2}$. This relation can be interpreted as a difference equation with the initial conditions $B_{1}=2 z \varepsilon, B_{2}=\varepsilon^{2}\left(4 z^{2}-1\right)$, whose solution is of the form

$$
B_{N}=\varepsilon^{N} U_{N}(z)
$$

where $U_{N}(z)$ is a second-kind Chebyshev polynomial. Taking Eq. (16) and the expression for $U_{N}(z)$,

$$
U_{N}(z)=\frac{\left(z+\sqrt{z^{2}-1}\right)^{N+1}-\left(z-\sqrt{z^{2}-1}\right)^{N+1}}{2 \sqrt{z^{2}-1}}=\frac{(1 / t)^{N+1}-t^{N+1}}{1 / t-t},
$$

where $t=z-\sqrt{z^{2}-1}$, into account, we obtain

$$
\operatorname{det}\left(Q_{N}-\rho I_{N}\right)=2 \varepsilon^{N}\left[\frac{t^{2 N+1}+1}{2 t^{N}}+\left(\frac{\alpha-\beta}{\varepsilon}\right) \frac{1-t^{2 N}}{\left(1-t^{2}\right) t^{N-1}}-(-1)^{N}\right]
$$

from Eq. (15). Hence, the characteristic equation for map differential (12) can be expressed as

$$
t^{2 N+2}+2\left(\frac{\alpha-\beta}{\varepsilon}\right) t^{2 N+1}-t^{2 N}-2(-1)^{N} t^{N+2}++2(-1)^{N} t^{N}+t^{2}-2\left(\frac{\alpha-\beta}{\varepsilon}\right) t-1=0 .
$$

It is now easy to calculate $\rho_{s}$ by finding the roots of Eq. (17) numerically. Comparing the $\rho_{s}$ values to unity for all possible values of the parameters $\alpha, \beta$, and $\varepsilon$, it is straightforward to determine the domains $D_{1}$ and $D_{2}$ of qualitatively different dynamics for an annular chain of diffusively connected maps with a single defect. The sections of these domains by the planes $\delta=$ const (where $\delta=\beta-\alpha$ ) are shown in Fig. 6. Estimate (13) for the regular-dynamics domain is shown by the dashed line. In this case as it was for the model with a periodic inhomogeneity, this domain (for positive $\delta$ ) is broader than that predicted by the estimate. However, in contrast to the periodic inhomogeneity, the estimate for $\delta<0$ fully coincides with the numerical result and also with the result for a homogeneous chain. Therefore, if the parameter corresponding to one of the system elements is smaller than those corresponding to all other elements, then this defect has no influence on the dynamics of the entire ensemble. However, if the anomalous parameter value is larger than the normal value, then the regular dynamics domain of the model is narrower than that in the homogeneous case. 


\section{Conclusion}

We investigated the behavior of systems of diffusively concatenated one-dimensional maps that model a one-dimensional active medium with a spatial inhomogeneity. Different types of inhomogeneity were examined. For all systems whose spatial inhomogeneity is related to the presence of two types of elements that differ by a parameter value, a lower estimate for the regular ensemble dynamics domain in the space of the parameters was derived analytically. This estimate was based on the localization of the differential eigenvalues of the map. Numerical and exact analytic results were obtained for two specific models of this class.

The results for the three examined cases, i.e., estimate (13) of the regular-dynamics domain for the class of models with two types of elements, the exact results for the system with periodic inhomogeneity (Fig. 3), and the exact results for the single-defect model (Fig. 6), reveal that there exist two general types of system dynamics. For the first type, the parameter of one sort of elements belongs to the regular-dynamics domain while the parameter of the other sort belongs to the unstable-behavior domain, and the general dynamics remains regular. For the second type, in contrast, the model dynamics can be unstable or, in the case of finite motion, chaotic. The realization of either behavior type depends on the diffusion parameter $\varepsilon$ and is possible only if the parameter is small. Obviously, the general dynamics of all chains belonging to the class of our interest cannot be regular for sufficiently large values of $\varepsilon$.

As far as we know, this work is the first attempt to describe the dynamics of inhomogeneous systems of concatenated maps analytically. The present knowledge is far from exhaustive even for the homogeneous discrete models of continuous media. Nuch is yet to be elucidated, many qualitative and quantitative questions remain open. Moreover, this work did not address the problems of possible stationary states of the model in the regularity domain, of wave motions along the chain and their dependence on the defects, of the dynamics in the case of time-dependent parameters, etc. Some of these questions will be answered in the near future $[23,24]$.

Acknowledgments. This work was supported in part by INTAS (Grant No. 97-1094).

\section{REFERENCES}

1. K. Kaneko, Physica D, 34, 1-41 (1989).

2. K. Kaneko, Physica D, 37, 60-82 (1989).

3. L. A. Bunimovich and Ya. G. Sinai, "Statistical mechanics of coupled map lattices," in: Theory and Applications of Coupled Map Lattices (K. Kaneko, ed.), Wiley, San Francisco (1993), pp. 169-189.

4. A. S. Mikhailov and A. Yu. Loskutov, (haos and Noise, Springer, Berlin (1996).

5. M. Bär and M. Eiswirth, Phys. Rev. E, 48, 1635-1637 (1993).

6. Zhilin Qu, J. N. Weiss, and A. Garfinkel, Phys. Rev. Lett., 58, 1378-1390 (1997).

7. S. Morita, Phys. Lett. A, 226, 172-178 (1997).

8. A. P. Muñuzuri, V. Perez-Muñuzuri, M. Gomez-Gesteira, L. O. Chua, and V. Perez-Villar, Int. J. Bifurcation Chaos Appl. Sci. Eng., 5, No. 1, 17-50 (1995).

9. L. A. Bunimovich, Physica D, 86, 248-255 (1995).

10. K. Kaneko, ed., Theory and Applications of Coupled Map Lattices, Wiley, San Francisco (1993).

11. Chaos Focus Issue on Coupled Map Lattices, Chaos, 2, No. 3 (1992).

12. K. Kaneko, Physica D, 41, 137-172 (1990).

13. L. A. Bunimovich and Ya. G. Sinai, Nonlinearity, 1, 491-504 (1988).

14. G. Perez, S. Sinha, and H. Cerdeira, Phys. Rev. E, 54, 6936-6939 (1996).

15. W. Just, J. Stat. Phys., 79, 429-449 (1995).

16. K. Kaneko, Physica D, 54, 5-19 (1991). 
17. V. S. Afraimovich, V. I. Nekorkin, G. V. Osipov, and V. D. Shalfeev, Stability, Structures and Chaos in Nonlinear Synchronization Nets [in Russian], Inst. Appl. Phys., Acad. Sci., Gor'kiy (1989).

18. V. A. Vasil'ev, Yu. M. Romanovskii, and V. G. Yakhno, Autowave Processes [in Russian], Nauka, Moscow (1987); English transl.: V. A. Vasil'ev, Yu. M. Romanovskii, D. S. Chernavskii, and V. G. Yakhno Autowave Processes in Kinetic Systems: Spatial and Temporal Self-Organization in Physics, Chemistry, Biology, and Medicine, Kluwer, Dordrecht (1987).

19. A. V. Gaponov-Grekhov, ed., Autowave Processes in Systems with Diffusion [in Russian] (Collected papers), Inst. Appl. Phys., Acad. Sci., Gor'kiy (1981).

20. Ya. B. Zel'dovich, G. I. Barenblatt, and V. B. Librovich, Mathematical Theory of Combustion and Explosion [in Russian], Nauka, Moscow (1980).

21. D. G. Aronson and H. F. Weinberger, "Nonlinear diffusion in population genetics, combustion, and nerve pulse propagation," in: Partial Differential Equations and Related Topics (J. A. Goldstein, ed.) (Lect. Notes Math., Vol. 446), Springer, Berlin (1975), pp. 5-49.

22. P. A. Horn and C. R. Johnson, Matrix Analysis, Cambridge Univ. Press, Cambridge (1986).

23. A. Yu. Loskutov, S. D. Rybalko, and D. N. Udin, "Dynamics of an inhomogeneous net of nonautonomous diffusively connected piecewise-linear maps," Regular and Chaotic Dynamics (forthcoming).

24. A. Loskutov, S. D. Rybalko, D. N. Udin, and N. V. Brillianton, "Space-time chaos and order in nonuniform coupled map lattices," Physica $D$ (submitted). 